WASHINGTON, D.C.-Prospects for soon beginning a gene-transfer clinical trial, which is designed to treat young individuals with severe combined immunodeficiency disease (SCID), are now somewhat dimmed because an alternative enzyme replacement treatment for the disease was recently approved by the Food and Drug Administration (FDA, Bethesda, MD).

Researchers at the National Institutes of Health (NIH, Bethesda, MD), who are conducting the gene-transfer experiment in humans (Bio/Technology 7:205, Mar. '89), returned to the NIH Recombinant DNA Advisory Committee (NIHRAC) late in March to propose an expansion of that study and also a new, limited clinical genetherapy test. While committee members recommended expanding the gene-transfer test, they greeted the new proposal with a mix of care and skepticism, deferring any decision on it at least until June.

The NIH researchers, led by $R$. Michael Blaese and W. French Anderson, propose to introduce the gene for the enzyme adenosine deaminase (ADA) into lymphocytes of certain SCID patients. SCID is an extremely rare genetic disorder, affecting about 40 children worldwide each year. In almost half the studied SCID patients, the ADA gene is defective, leading to the absence of that enzyme. The biochemical defect in turn renders such an individual's immune system ineffectual, leading to exquisite susceptibility to infections.

Current therapies for treating individuals with SCID include extraordinary isolation measures to prevent exposure to infectious agents, or bone marrow transplantation (BMT) to introduce cells carrying the $\mathrm{ADA}$ gene. Although BMT can be very effective, it works only when an appropriate donor is available: usually only a sibling provides a close enough tissue match.

A third therapeutic approach became available in March when the FDA approved an enzyme replacement therapy for SCID based on use of purified ADA that is chemically bound to polyethylene glycol (PEG) carrier molecules (Bio/Technology 8:279, Apr. '90). The non-toxic PEG polymer prolongs the lifetime of the bound enzyme in the body. The product, which was granted orphan drug status by FDA, is made by Enzon (South Plainfield, NJ).

So far, 13 children have been treated this way, receiving weekly injections of the PEG-bound enzyme that could continue for the remainder of their lives. According to Enzon, all patients have shown clinical response to the drug, with no serious sideeffects. In addition, all have demonstrated an improved quality of life: some have been taken out of isolation and gone back to their families; others have been able to attend school or participate more in extracurricular activities.

With such alternatives to consider, members of NIHRAC have been carefully examining the proposed gene-replacement experiment, which raises complex safety and efficacy issues. The proposed test entails removing a SCID individual's lymphocytes and introducing the ADA gene into them. The engineered cells

\section{TREATING ADA IN EUROPE}

D. Valerio's group at the Radiobiological Institute TNO (Rijswijk, The Netherlands) is also developing gene therapy for $\mathrm{ADA}$. The view of $\mathrm{ADA}$ as a good model system, however, was challenged by Peter Waele of Innogenetics (Ghent, Belgium), speaking at a conference held in March in Amsterdam, the Netherlands (see "From Clone to Controversy," this issue).

Waele pointed out that the disease could be treated, albeit somewhat unsatisfactorily, with bovine adenosine deaminase. essarily involved children should weigh heavily in the choice of an experimental system.

In defense of ADA gene therapy, Valerio noted firstly that, 20 years ago, SCID patients were the first to undergo bone marrow transplant. He also pointed out that the process of $T$ cell extraction, infection, and reinsertion is less calamitous than bone marrow transplant.

-John Hodgson Furthermore, he believes that the fact that ADA treatment nec- would be reintroduced into the patient, where the ADA gene would presumably produce enzyme for several weeks. The entire procedure would then need to be repeated because the finite life cycle of lymphocytes requires replenishment.

Because children with SCID are so vulnerable to infections, the usual concerns involved in testing a new clinical procedure are considerably amplified. For instance, the potential presence of infectious contaminants or the viral vectors used for manipulating the ADA gene before it is introduced into the lymphocytes of patients could be life-threatening-at least in a worst-case analysis. Thus, NIHRAC members and other participants are insisting on an unusually detailed examination of such issues and the precautions being taken before approving the clinical test. They also say they are troubled that animal experiments in mice and primates have not provided clear and weighty evidence in favor of proceeding clinically.

Beyond that, the availability of the Enzon PEG-bound enzyme product further restricts the NIH research team. For medical and ethical reasons, any SCID patients who will be candidates for the gene-therapy test will be receiving the FDA-approved treatment. The availability and use of this product, however, could cloud evidence indicating whether the experimental gene therapy is working. On the other hand, Enzon president Abraham Abuchowski points out that, "without PEG-ADA, potential patients [for the gene-therapy regimen] would not have enough lymphocytes to be treatable." Eventually, if the gene therapy approach received a go-ahead and then appeared to be working, the patients might be weaned from PEG-ADA.

Members of the NIH research team appear frustrated by the elaborate, multi-layered reviews required by NIH and FDA before the clinical tests can begin. However, members of NIHRAC say that the committee has adjusted its schedule to move as quickly as possible while still adhering to federal procedures for notifying the public and inviting its participation in the review. While the researchers prepare a "full write-up," NIHRAC will "tiptoe for a while in this new area," says one committee member. "Unless NIHRAC procedures are followed in detail, it could cost all of us more time."

-Jeffrey L. Fox 